\title{
Poriferan survivin exhibits a conserved regulatory role in the interconnected pathways of cell cycle and apoptosis
}

\author{
B Luthringer ${ }^{1}$, S Isbert ${ }^{1}$, WEG Müller ${ }^{1}$, C Zilberberg ${ }^{1}$, NL Thakur ${ }^{1}$, G Wörheide ${ }^{2}$, RH Stauber ${ }^{3}$, M Kelve ${ }^{4}$ and M Wiens ${ }^{\star, 1}$
}

Survivin orchestrates intracellular pathways during cell division and apoptosis. Its central function as mitotic regulator and inhibitor of cell death has major implications for tumor cell proliferation. Analyses in early-branching Metazoa so far propose an exclusive role of survivin as a chromosomal passenger protein, whereas only later during evolution a complementary antiapoptotic function might have arisen, concurrent with increased organismal complexity. To lift the veil on the ancestral function(s) of this key regulator, a survivin-like protein (SURVL) of one of the earliest-branching metazoan taxa was identified and functionally characterized. SURVL of the sponge Suberites domuncula shares considerable similarities with its metazoan homologs, ranging from conserved exon/intron structure to presence of protein-interaction domains. Whereas sponge tissue shows a low steady-state level, SURVL expression was significantly upregulated in rapidly proliferating primmorph cells. In addition, challenge of tissue and primmorphs with heavy metal or lipopeptide stimulated SURVL expression, concurrent with the expression of a newly discovered caspase. Complementary functional analyses in transfected HEK-293 cells revealed that heterologous expression of a SURVL-EFGP fusion not only promotes proliferation but also enhances resistance to cadmiuminduced cell death. Taken together, these results suggest both a deep evolutionary conserved dual role of survivin and an equally conserved central position in the interconnected pathways of cell cycle and apoptosis.

Cell Death and Differentiation (2011) 18, 201-213; doi:10.1038/cdd.2010.87; published online 23 July 2010

Apoptosis has a crucial role not only in countless physiological processes (e.g., elimination of redundant cells during vertebrate embryonic development) but also during pathogenesis. As apoptosis relies on the clear-cut function of numerous proand antiapoptotic factors, any malfunction on their part or disruption of their respective interplay inevitably leads to dysfunctional cellular metabolism. The ensuing delayed or premature cell death often manifests in severe pathologies, including carcinogenesis and neurodegenerative diseases.

The groundbreaking discovery of the Caenorhabditis elegans proteins cell death abnormality (Ced)- 9 , homolog of human antiapoptotic Bcl-2, and Ced-3, homolog of human caspase-1, has shown the molecular conservation of the apoptotic cell death program along a broad range of Metazoa, from nematodes to vertebrates. ${ }^{1}$ Since then, the fundamental role of apoptosis has been increasingly recognized in various physiological processes. In addition, several invertebrate model organisms revealed a significant potential for analyses of apoptotic mechanisms, not only under an evolutionary point of view. Thus, the identification of the human crumbs homolog 1 and its causative role in the manifestation of retinitis pigmentosa was based on the discovery of mutational variants of the Drosophila melanogaster protein crumbs. ${ }^{2}$ In addition, loss-of-function mutations of the $C$. elegans dystrophin-1 gene confirmed the role of human dystrophin in the manifestation of the neuromuscular disease of Duchenne. ${ }^{3}$ During the last years a novel invertebrate model system has emerged: sponges (phylum Porifera), which are among the earliest-branching nonbilaterian animals. ${ }^{4}$ Recent biomarker analyses date the occurrence of probable stem-group poriferans back before the end of the Marinoan glaciation $(\sim 635 \mathrm{Myr} \text { ago })^{5}$ and molecular clock analyses estimated that the main poriferan lineages diverged in the Cryogenian/ Ediacaran, ${ }^{6}$ well before the rapid appearance of major metazoan groups (Cambrian Explosion).

Till today, the model system 'Porifera' has revealed several characteristics that have proven valuable for evolutionary studies within a comparative context. The major avail is the phylogenetically basal position of Porifera allowing a unique vantage point on the hypothetical common ancestral taxon, the Urmetazoa. Moreover, comparative transcriptomics and proteomics divulged several examples of poriferan molecules, genetic features, protein domains, among others, that remained conserved throughout evolution, but otherwise

\footnotetext{
${ }^{1}$ Institute for Physiological Chemistry and Pathobiochemistry, Johannes Gutenberg University, Medical School, Mainz; Germany; ${ }^{2}$ Department of Earth and Environmental Sciences, Ludwig-Maximilians Universität, München; Germany; ${ }^{3}$ Department of Otorhinolaryngology, Molecular and Cellular Oncology, Medical School, Johannes Gutenberg University, Mainz; Germany and ${ }^{4}$ Department of Gene Technology, University of Technology, Tallinn; Estonia

${ }^{*}$ Corresponding author: M Wiens, Institute for Physiological Chemistry and Pathobiochemistry, Johannes Gutenberg University, Medical School, Duesbergweg 6 , D-55099 Mainz; Germany. Tel: + 496131392 5961; Fax: + 496131392 5243; E-mail: wiens@ @uni-mainz.de

Keywords: survivin; IAP; caspase; sponges; Suberites domuncula; cadmium

Abbreviations: aa, amino acid; Bcl-2, B-cell lymphoma-2; BIR, baculoviral IAP repeat; CDE, cell-cycle dependent elements; Ced-9/-3, cell death abnormality -9/-3; CHR, cell-cycle homology regions; CPC, chromosomal passenger complex 1; DIG, digoxigenin; ELISA, enzyme-linked immunosorbent assay; IAP, inhibitors of apoptosis protein; LMB, leptomycin B; LSM, laser scanning microscopy; ML, maximum likelihood; MTT, 3-(4,5-dimethylthiazol-2-yl)-2,5-diphenyltetrazolium bromide; NES, nuclear export signal; NJ, neighbor-joining; ORF, open reading frame; PBS, phosphate-buffered saline

Received 17.6.09; revised 25.5.10; accepted 17.6.10; Edited by M Hengartner; published online 23.7.10
} 
have been lost in invertebrate model systems traditionally used, $C$. elegans and $D$. melanogaster. ${ }^{7}$ Furthermore, the majority of poriferan genes show a closer sequence similarity to their human homologs than to the respective molecules of worm and fly (similar to some anthozoans), probably caused by an accelerated evolution of the latter animal lineages. ${ }^{8,9}$

The fine-tuned balance between apoptosis and proliferation is upheld by numerous regulatory molecules that maintain tissue integrity and, ultimately, ensure survival of the organism. The inhibitors of apoptosis (IAP) family comprises structurally related proteins (the presence of at least one baculoviral IAP repeat (BIR) domain is mandatory) that were originally discovered in baculovirus. In addition to their cell death regulating activities, several IAP have a complementary role in cell division, in particular attributed to survivin. Accordingly, the dual role of human survivin is based on its function as (i) a chromosomal passenger protein that controls segregation of chromatin/regulation of cytokinesis and (ii) a caspase inhibitor that prevents processing/activation of caspases. ${ }^{10}$ As transcription from its single gene locus is upregulated in most of the tumor cell types, survivin is a wellestablished analytical marker of neoplasms. ${ }^{11,12}$ The function of survivin homologs in nonbilaterian Metazoa is much less well-defined. Accordingly, similar to vertebrate survivin, the $D$. melanogaster homolog deterin deters cells from caspaseinduced apoptosis and some studies propose an additional pro-proliferative effect. ${ }^{13}$ On the other hand, the $C$. elegans survivin homolog BIR-1 seems to be exclusively involved in cytokinesis, in particular during the embryogenesis. ${ }^{14}$

In this study, we report the discovery and functional characterization of a survivin-like protein (SDSURVL) from a member of the ancestral-like clade Porifera, Suberites domuncula. SDSURVL shows both significant sequence homologies to human survivin (in particular concerning the BIR domain) and a conserved genetic structure. Complementary studies in vivo and in transfected cells suggest a conserved dual role of survivin and, concurrently, indicate a loss of function in certain rapidly evolving and highly adapted metazoan taxa.

\section{Results}

Characterization of the poriferan survivin-like protein SDSURVL. The complete SDSURVL cDNA was isolated from a $S$. domuncula cDNA library by polymerase chain reaction $(P C R)$ and degenerate primers that were directed against a conserved region within the BIR domain. SDSURVL consists of $620 \mathrm{nt}$ (excluding the poly(A) tail) that encompass an open reading frame (ORF) of $447 \mathrm{nt}$ (excluding the first stop codon), beginning at $\mathrm{nt}_{43-45}$ (Met start $_{\text {. }}$. During northern blot analyses, a digoxigenin (DIG)-labeled probe detected a transcript whose size was consistent with that of the cDNA ( $\approx 650 \mathrm{nt}$; Figure $4 \mathrm{a})$. The deduced protein, SDSURVL, comprises 149 amino acid (aa) with an expected size of $17003 \mathrm{Da}$. Analyses of SDSURVL revealed significant sequence similarity to vertebrate survivin homologs, such as Gallus gallus survivin isoform 1 (with an expect value ( $E$-value) ${ }^{15}$ of 2 e-20; $32 \%$ identical and $52 \%$ similar aa) and human survivin (8 e-18; 30/49\%) (Figure 1a). Considerably lower was the similarity to survivin homologs of more basal Metazoa and fungi, for example, $D$. melanogaster deterin (7 e-11; 45/24\%), placozoan (Trichoplax adhaerens) survivin-like protein (2 e-11; 19/35\%), and Schizosaccharomyces pombe survivin homolog (5 e-11; 34/48\% with respect to the BIR domain $\left.\left(\mathrm{aa}_{117-194}\right)\right)$. Viral genomes often encode antiapoptotic or cell-cycle promoting proteins in order to bypass the host defense system. Viral survivin-like proteins generally show lower sequence homology to their metazoan counterparts and in particular to $S$. domuncula survivin, for example, Epiphyas postvittana nucleopolyhedrovirus IAP-3 (4 e-07; $17 \%$ aa similarities) and Cydia pomonella granulosis virus ORF17 IAP-3 (3 e-06; 16\%).

As SDSURVL features a single BIR domain, a putative nuclear export signal (NES), and a coiled-coil region, but is missing a RING domain (characteristic of non-survivin IAP), the molecule represents a novel member of the survivin subfamily of IAP. The poriferan BIR domain $\left(\mathrm{aa}_{20-93}\right)$ has an $E$-value of $4.85 \mathrm{e}-22$ and is consistent with the Prosite consensus sequence PS01282 with two exceptions (marked in bold): (HKEPILVY)-x(2)-R-x(3,8)-(FYW)-x(11,14)-(STAN)G-(LMF)-x-(FYHDA)-(x(4) $x(7))-($ DESL)-x(2,3)-C-x(2)-C-x(6) -(WA)-x(9)-H-x(4)-(PRSD)-x-C-x(2)-(LIVMA). The putative NES (aa $\left.a_{95-103}\right)$ loosely fits a consensus sequence proposed by, Heger et al. ${ }^{16}$ L-x(2,3)-(FILVMP)-x(2)-(LI)-x-(LIV). Furthermore, COILS algorithms indicate the typical $\mathrm{COOH}$ terminal coiled-coil conformation $\left(\mathrm{aa}_{127-147}\right)$, usually present in survivin but missing in other IAP family members. Moreover, the conserved threonine residue, whose phosphorylation by $\mathrm{p} 34 \mathrm{cdc} 2$ mediates human survivin activation, ${ }^{17}$ is present in SDSURVL $\left(\mathrm{aa}_{26}\right)$. In addition, a ubiquitin ligase complex-binding destruction motif (APCC-D box; $a_{136-141}$ ) was predicted.

\footnotetext{
Figure 1 Suberites domuncula survivin-like protein SDSURVL. (a) The deduced amino acid sequence (SUBDO) was aligned with survivin homologs of $D$. melanogaster (DROME, NCBI accession number NP_650608), Anopheles gambiae (ANOGA, XP_317026), Nematostella vectensis (NEMVE, XP_001624746), human (HUMAN, CAG46540), G. gallus (CHICK, NP_001012318), Danio rerio (DANRE, NP_919378), and S. pombe (SCHPO, NP_587866). Conserved residues (identical or similar with respect to physicochemical properties) in all sequences are shown in white letters on black; those in $80 \%(60 \%)$ are in white on gray (black on light gray). The following features are marked, BIR domain, NES, coiled-coil region, APCC-D box (underlined) as well as the putative phosphorylation site (star). (b) Composite consensus tree of the phylogenetic analyses, depicting the evolutionary relationship of $S$. domuncula SDSURVL to survivin homologs of Metazoa, fungi, and viruses. The tree was generated after alignment of the aforementioned sequences, further integrating sequences from: T. adhaerens (TRIAD, EDV24865), Schistosoma japonicum (SCHJA, AAW27178), Felix catus (FELCA, NP_001009280), mouse (MOUSE, NP_033819), Xenopus laevis (XENLA, BAD98266), Pan troglodytes (PANTR, XP_512010), Sus scrofa (PIG, NP999306), Canis lupus (CANFA, NP_001003348) as well as BIR-containing viral sequences of Helicoverpa armigera nuclear polyhedrosis virus (NPVHA, NP_075172), Buzura suppressaria NPV (NPVBS, AAC34373), Epiphyas postvittana NPV (NPVEP, NP_203195), Choristoneura fumiferana NPV (NPVCF, NP_848342), Amsacta moorei entomopoxvirus (AMEPV, NP_064803), and Cydia pomonella granulosis virus (GVCP, NP_148801). Close to each node are the bootstrap values in the order, NJ, ML, and the posterior probability estimates of the Bayesian inference
} 
DROME ANOGA NEMVE HUMAN CHICK DANRE SUBDO SCHPO

DROME ANOGA NEMVE HUMAN CHICK DANRE SUBDO SCHPO

DROME ANOGA NEMVE HUMAN CHICK DANRE SUBDO SCHPO
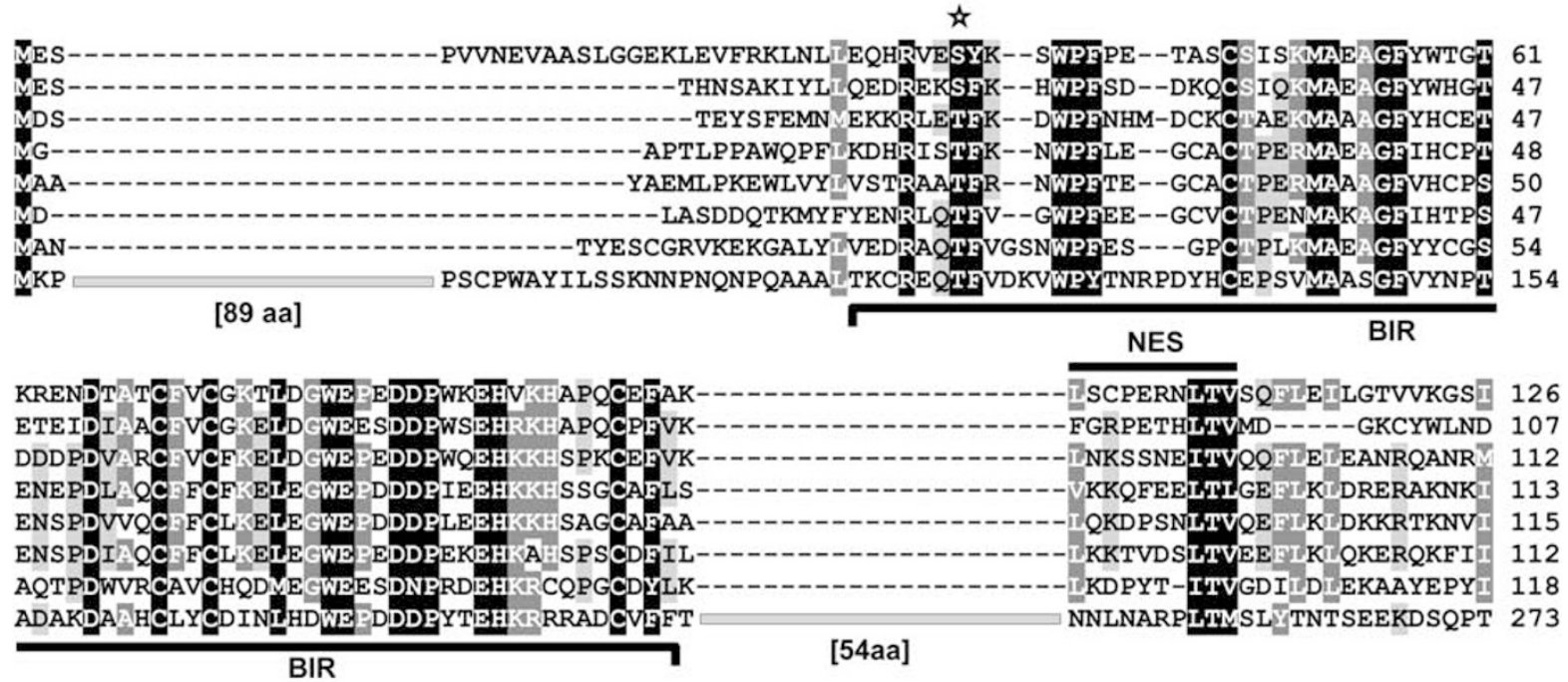

EKTCKAFKSSFVRENEKRLDEFTRNQK-

EEKIKLTDITFALN--LOCDEFVTIMMAT---

RKYVDANLKDFEHMRDQAREEMMKLVPEP-_-

AKETNNKKKEFEETVKKVRRAIEQIAAMD-----

KKAISQKETDIEDVAKGVRHAIENMGP--

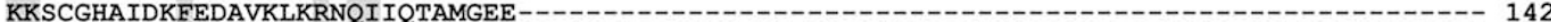

RQEGDKLSADLDEMVGDMREQLVNAEKTRTS-

RAPQSPTKPVLLTAPRRKNKSPKKSKPAVFKPVKPIFSDEDEDDDDL

$$
\text { Coiled-coil region }
$$$$
\text { APCC Dbox }
$$

b

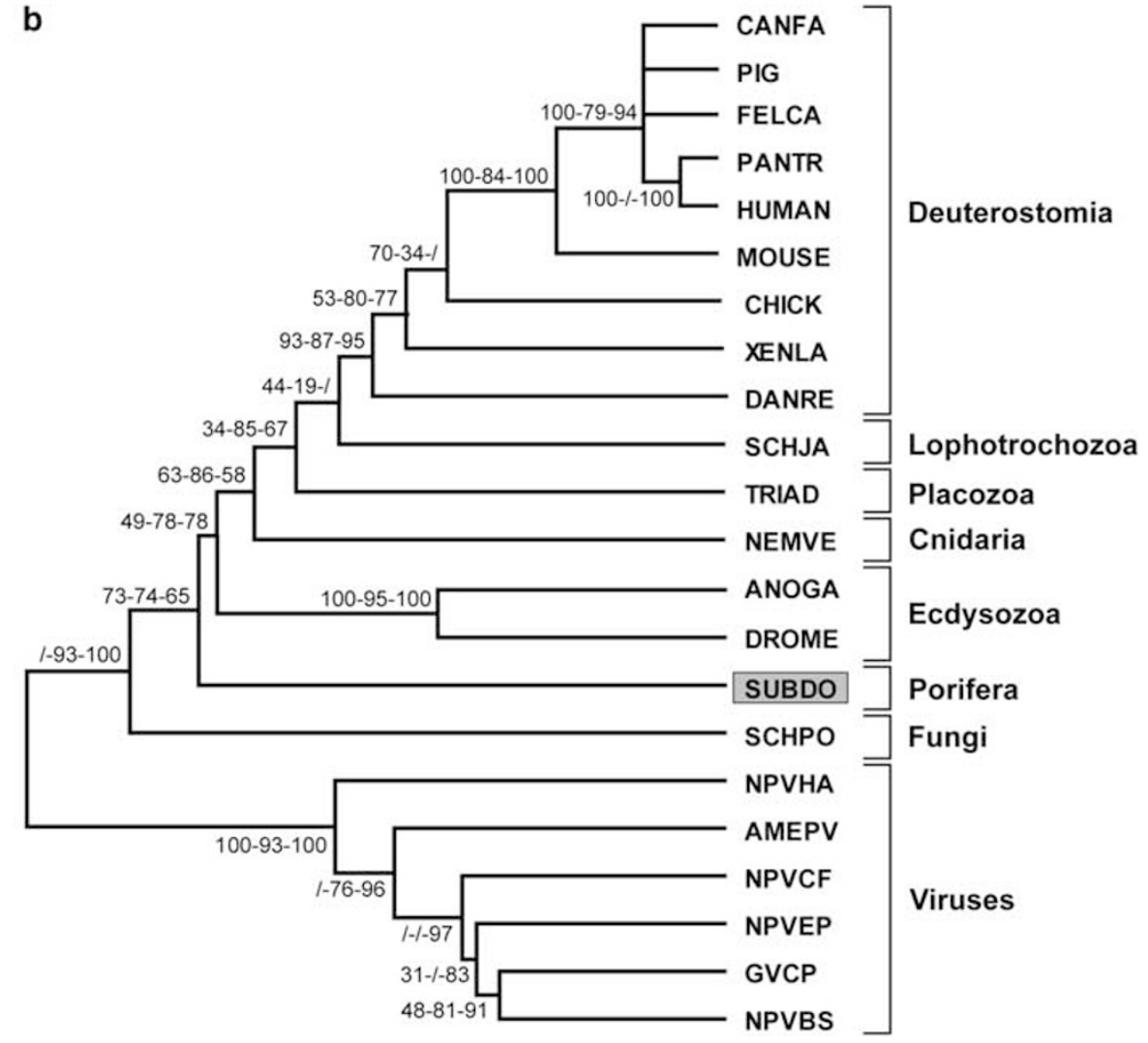

CANFA

PIG

FELCA 
To determine the phylogenetic relationship of survivin-like proteins, molecular phylogenetic analyses were carried out by neighbor-joining (NJ), maximum likelihood (ML), and Bayesian methods, including the so far known sequences of metazoan taxa as well as related BIR-containing sequences of viruses and yeast. Accordingly, the resulting composite consensus tree shows the poriferan SDSURVL protein at the base of a monophyletic metazoan clade, whereas viral sequences form a separate monophyletic group (Figure 1b).

Characterization of the poriferan survivin-like gene (SDSURVL). The $S$. domuncula gene comprises four exons and three introns (Figure 2a). Each exon/intron border shows typical splicing signals (GT-intron-AG). The phases of introns vary: the first and third introns are in phase 0 , whereas the second intron is in phase 2. The human survivin gene shares the same features (Figure $2 b$ ) except for the borders of the third intron (AT-intron-GG). Parts of the first and third exons and the complete second exon are encoding the BIR domain (Figure 2c). Analyses of the $5^{\prime}$ flanking region (Figure 2d) of human (1) and murine (2) survivin genes reveal the presence of a TATA-less promoter, containing a canonical $\mathrm{CpG}$ island and several transcription factor-binding sites. ${ }^{18}$ However, in silico analyses of the putative poriferan promoter region (3) predicted the presence of a TATA box ( $\mathrm{nt}_{-64}$ to -73 ) in addition to other cis-regulatory elements, CAAT box ( $\mathrm{nt}_{-109}$ to -113 ), and ribosomalbinding site $\left(\mathrm{nt}_{-5}\right.$ to -8 ). Moreover, similar to the human and murine promoter two putative Sp1 sites (nt ${ }_{-51},{ }_{-292}$ ) have been predicted, although in contrast to the former ones no cell-cycle dependent elements (CDE) or cell-cycle homology regions $(\mathrm{CHR})$ have been found in the poriferan non-coding region.

\section{Characterization of the poriferan caspase-like protease 2 (SDCASL2). Caspases (cysteine-dependent aspartyl-} specific proteases) are synthesized as zymogens that become activated by scaffold-mediated transactivation or by cleavage using upstream proteases. ${ }^{19}$ Since the antiapoptotic properties of vertebrate survivin have been functionally linked to inhibition of caspase-7 and -3 , a $S$. domuncula cDNA library was screened for homologous molecules. The correct size of the resulting clone, SDCASL2, then was verified on northern blots (Figure 4a). SDCASL2 $(1,827 \mathrm{nt}$, excluding poly $(\mathrm{A}))$ comprises an ORF between $\mathrm{nt}_{175-177}\left(\right.$ Met $\left._{\text {start }}\right)$ and $\mathrm{nt}_{1738-1740}$ (stop), coding for a putative protein of 521 aa that was termed caspase-like protease 2 (SDCASL2; calculated $\mathrm{Mr}$ 58,427). SDCASL2 revealed sequence homology not only to SDCASL ( $E$-value 7 e-31; $33 \%$ aa identical and $52 \%$ similar aa) but also to members of the caspase-7 subfamily, for example, Xenopus laevis and human representatives ( $E$-value 3 e-16 (21/33\%) and 3 e-14 (19/35\%), respectively) (Figure 3 ).

All caspases contain a CASc domain, which is cleaved into two subunits ( $1010, p 20)$ during activation. SDCASL2 represents a putative procaspase, comprising subunits p10 $\left(a_{191-267}\right)$ and p20 $\left(a_{22-154}\right)$, the latter containing the characteristic Cys and His active sites (Figure 3 ). The Cys active site consensus pattern is conserved (Prosite pattern PS01122) with two exceptions, marked in bold
((KL)-P-K-(LIVMF)-(LIVMFY)-(LIVMF)(2)-(QPD)-(AF)-C-(RQG)$(\mathrm{GE})$ ), whereas the histidine active site (PS01121) features three alterations, (HL)-x(2,4)-(SC)-x(1,2)-(A)-x-(LIVMFY)(2)(ST)-G-H-G.

The absence of a large $\mathrm{NH}_{2}$-terminal prodomain, characteristic of initiator caspases, suggests a classification to the group of effector caspases. However, similar to SDCASL, SDCASL2 carries an unusual long $\mathrm{COOH}$-terminal stretch of 254 aa, containing a putative double-stranded RNA-binding motif (aa $441-514$; PF0035 (Pfam)).

Evolutionary conserved function(s) of poriferan survivin. To elucidate the controversially discussed ancestral function of survivin (or if indeed its dual role is conserved through metazoan evolution), functional analyses were carried out in sponge tissue and primmorphs (aggregates of proliferating sponge cells). Moreover, functional conservation was assessed in a heterologous vertebrate cell model (SDSURVL-expressing transfected HEK-293 cells) that has been previously used to explore expression and functional mechanisms of survivin. ${ }^{20,21}$

Regulation of proliferation. Comparative northern blot analyses were carried out, showing an increasing level of SDSURVL transcripts during the first 2 weeks of primmorph growth, whereas the control (taken immediately after primmorph formation) revealed a considerably lower expression level (Figure 4a). After 14 days, survivin transcription reached the steady state. On the other hand, in adult sponge tissue survivin transcripts were barely detected. In addition, SDCASL2 expression was very low throughout the experiments, in both primmorphs and sponge tissue (Figure 4a). Subsequently, SDSURVL and SDCASL2 expression was investigated in aging primmorphs (2 weeks of age) by complementary in situ hybridization studies (Figure 4b). For this purpose, primmorph cryosections were incubated with labeled, single-stranded sense (control) or antisense probes. Both sense probes did not produce specific signals. In contrast, the antisense SDSURVL probe detected survivin transcripts abundantly and homogenously throughout the sections, whereas only few SDCASL2 copies were identified, mainly in the surface cell layer and in cells lining the aquiferous canal system within the mesohyl (internal part of the sponge) (Figure 4b).

In parallel, a construct encoding a fusion of SDSURVL and COOH-terminal EGFP (SDSURVL-EGFP) was introduced into HEK-293 cells. Expression was confirmed microscopically (Figure $5 \mathrm{~b}$ ) and by immunodetection, using anti-EGFP antibodies (Figure 8; expected size of $45 \mathrm{kD}$ corresponding to the SDSURVL-EGFP fusion). The transfection efficiency was determined by the percentage of EGFP-positive HEK-293 cells by fluorescence microscopy. An average transfection efficiency of $94.6 \%$ was calculated.

Subsequently, the putative regulatory function of SDSURVL in cytokinesis was explored by monitoring the concentrations of SDSURVL-EGFP-transfected (HEK293SDSURVL) and mock-transfected HEK-293 cells, $48 \mathrm{~h}$ after transfection (Figure 5a). Whereas mock-transfection provoked no differences in cell concentration compared with the nontransfected control, SDSURVL-EGFP expression caused a 2.3-fold increase in the number of HEK293-SDSURVL cells 
a ATGGCTAATACGTATGAGAGTTGTGACAGAGTAAAGGAGAAGGGAGCGCTCTATCTTGTGGAGGACAGAGCACAAACGTTCGTTGGAAGTAATTGGCCT Met

TTTGAGAGTGGGCCATGCACTCCTCTGAAAGTAAGATGATTGGACATCCAGTGTTCATGTCAGAGTGTTGGATGTGAGTCATGCAATGGTTTGTTTGCT CTGTTGTTUAGATGGCAGAGGCTGGTTCTATTATTGTGGCAGTGCTCAAACTCCAGACTGGGTTCGTTGTGCTGTGTGTCATCAAGACATGGAGGTT GGGAAGAATCAGACAATCCCCGGTATGTGTATGTTGTAAATATTGAAATTGTCCTTGAGTTGGCTAAATCTACACGTTIAGTATGAACACAAGCGTTGT CAGCCTGGATGTGATTATCTCAAATTGAAAGACCCGTACACCATCACTGTGGGGGACATCTTAGACTTGGAGAAGGCAGCGTATGAGCACTATATIGTF $\frac{\text { AGTGACAGATAGTTTTGACTGAACATATAAAGCTATAATCGCTGTTTTCTTTTMGAGACAAGAGGGTGACAAGTTGTCTGCAGATCTAGATGAGATGGT }}{\text { Intron } 3}$

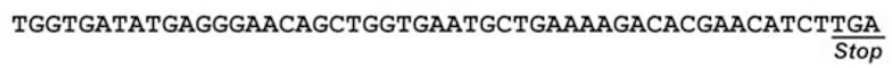

b SURV_HUMAN ------ATGGGTCCCCGACGTTGCCCCTGCCTGGCAGCCCTTT--CTCAAGGACCACCGCATCTCTACATTCA--AGAAT 73 SDSURVL_SUBDO ATGGCTAATACGTATGAGAGTTGTGACAGAGTAAAGGAGAAGGGAGCGCTCTATCTTGTGGAGACAGAGCACAAACGTTCGTTGGAAGT 90

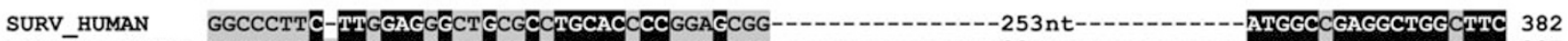

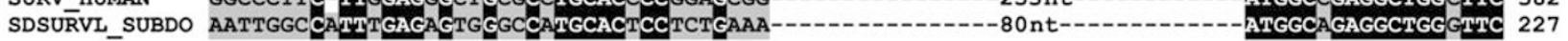

SURV_HUMAN ATCOACTECCCCACTEAGAACGAGCCAGACTTGGCCCAGTGTTTCTTCTECTTCAAGGAGCTGGAAGGCTGGGAGCOAGATGACGACCCC 472 SDSURVL_SUBDO TATTATTGTGGCAGTGCTCAAACTCCAGACTGGGTTCGTTGTGCTGTGTGTCATOAAGACATGGAGGGTTGGGAGAATCAGACAATCCC 317

SURV_HUMAN AT----------1875nt-------AGAGGACATAAAAGCATTCETCCGGTTECECTTTCCTTTCTGTCAAGAAGCAGTTTGA 2409 SDSURVL_SUBDO CG----------58nt---------TGATGAACACAAGCGTTGICAGCCTGGATGTGATTATCTCAAATIGAAAGACCCGT---A 434

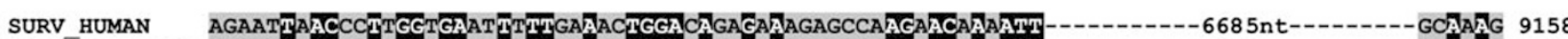

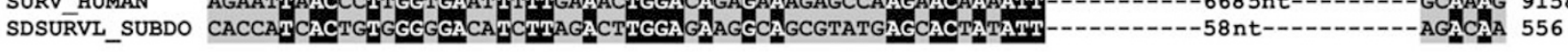
SURV_HUMAN GAAACCAACAATAAGAAGAAAGATTTGAGAAACTGCGGAGAAAGTECGCCGT--GCCATCGAGCAGCTGGCTECCATGGA----TTGA 9242 SDSURVL_SUBDO GAGGTGACAAGTTGTCTGCAGATCTAGATGAGATGGTTGGTGATATEAGGGACAGCTGGTGATGCTGAAAAGACACGAACATCTTGA 646

C

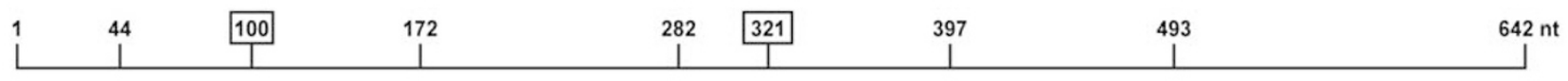

\begin{tabular}{|l|l|l|l|l|l|}
\hline & Exion 1 & Exon 2 & Exon 3 & Exon 4 & \\
\hline
\end{tabular}

BIR

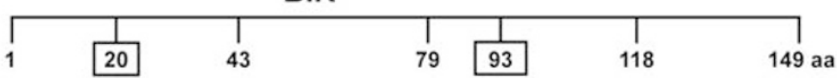

d

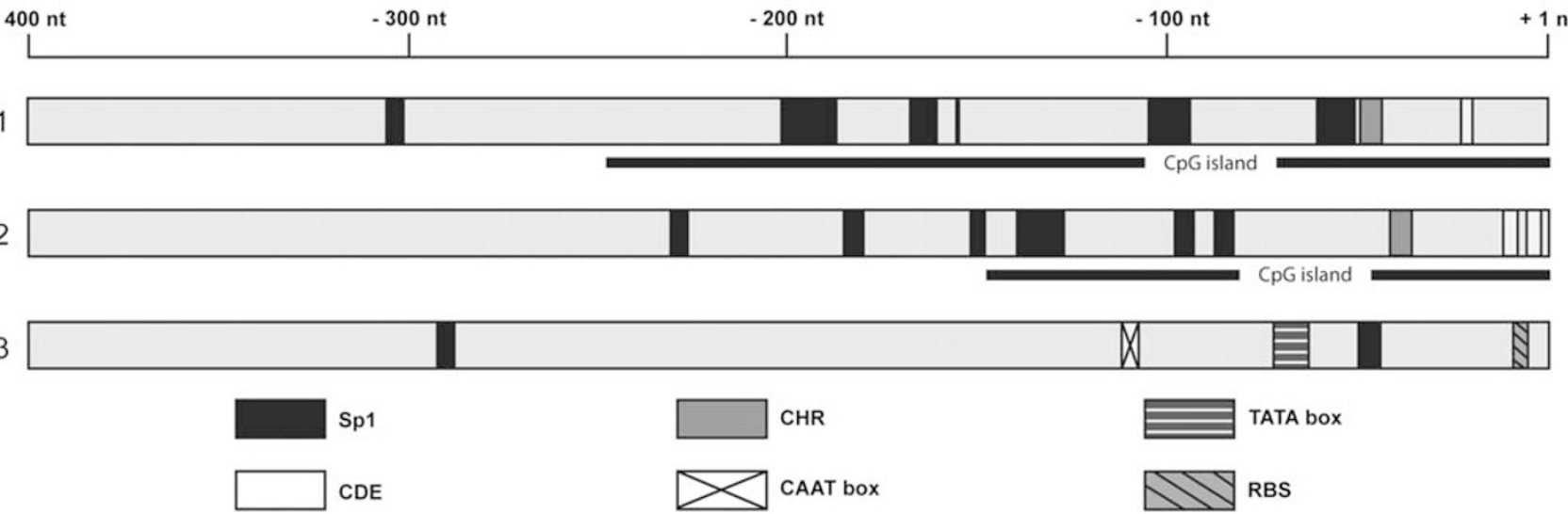

Figure 2 Suberites domuncula survivin-like gene. (a) Exon/intron architecture. Each exon/intron border is marked (boxed) as well as introns, start and stop codon (underlined). (b) Alignment of SDSURVL and human survivin exons with correlating intron phases. Sizes of introns are indicated. (c) Schematic representation of SDSURVL mRNA and its translational product, drawn to scale. The BIR domain and its contributing exons are marked, as well as untranslated regions. (d) Schematic representation of survivin promoter regions from representative species: murine (1), human (2), and poriferan (3). The different elements: Sp1 (sequence-specific DNA-binding protein-1), CDE (cell cycle dependent element), CHR (cell cycle homology region), CAAT and TATA boxes, and RBS (ribosomal-binding site) are drawn to scale (first base of Met start, $+1 \mathrm{nt}$ ). Regions of high $\mathrm{CpG}$ island density are also indicated. The promoter region is depicted up to $-400 \mathrm{nt}$ 


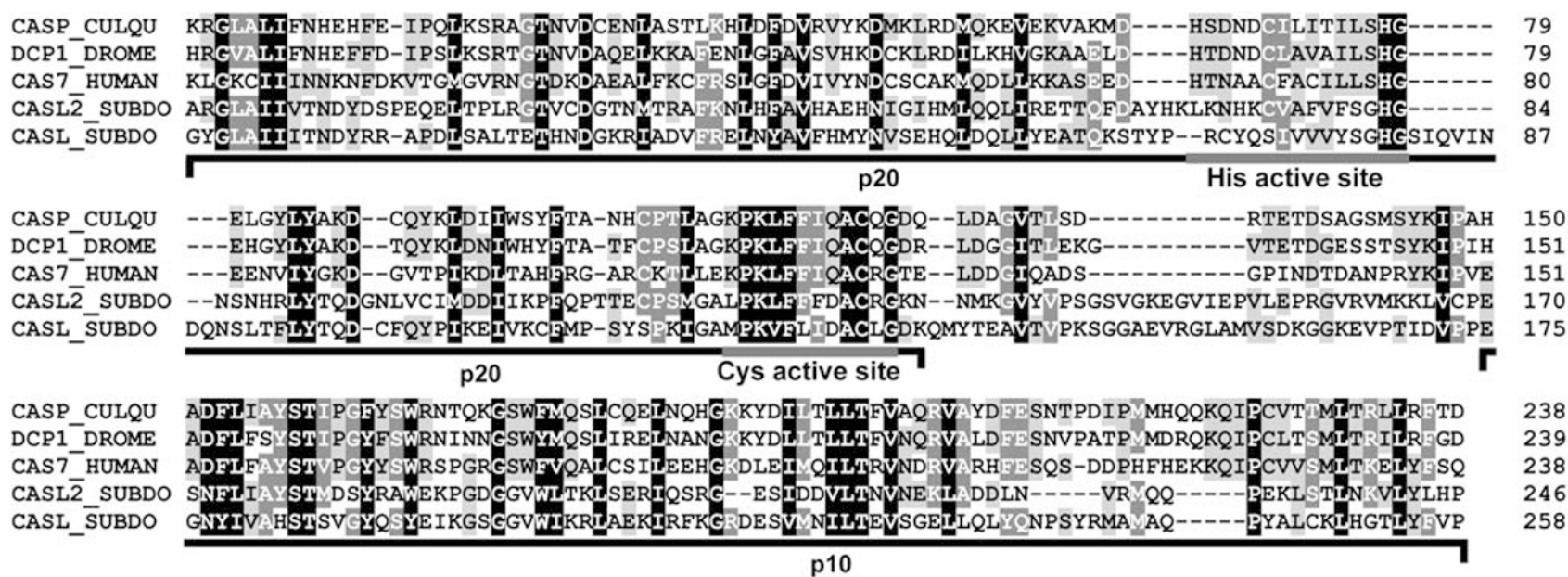

Figure 3 Suberites domuncula caspase-like protease 2 SDCASL2. The CASc domain of the deduced poriferan protein (CASL2_SUBDO; aa $22-267)$ was aligned with the corresponding domain of $S$. domuncula caspase-like protein (CASL_SUBDO; CAL36107; aa $17-274$ ); D. melanogaster DCP-1 (DCP1_DROME; AAB58237; aa $77-315$ ), Culex quiquefasciatus (CASP_CULQU; XP_001842236; $a_{60-304}$ ), and human caspase-7 (CAS7_HUMAN; BAG62964; aa ${ }_{74-311}$ ). The putative subunits contained within (p20 and p10) are marked as well as the Cys and His active sites

(significance level $P \leqslant 0.05)$. In addition, trypan blue staining did not show altered cell viability between control and SDSURVL-EGFP-expressing populations.

Finally, subcellular localization and nucleocytoplasmic trafficking of SDSURVL-EGFP in transfected cells was analyzed by confocal laser scanning microscopy (LSM). For this purpose, HEK293-SDSURVL cells were incubated with the nuclear export inhibitor leptomycin B (LMB) for 30 min or remained untreated (control). After counterstaining of dsDNA with the far-red fluorescent dye DRAQ5, LSM showed that in the controls SDSURVL-EGFP predominantly localizes in the cytoplasm and, to a lesser degree in the nucleus (Figure 6a). Consequently, the fluorescence pattern of the merge revealed a barely detectable colocalization of fusion (green) and nuclei (red). On LMB treatment, SDSURVL-EGFP accumulated in the nucleus. The merged channel clearly depicted a significant colocalization of nuclei and SDSURVL-EGFP (Figure 6b; yellow), whereas the staining intensity of cytoplasmic SDSURVL-EGFP is decreased, compared with the untreated control.

Regulation of apoptosis. To explore the putative implementation of SDSURVL as a prosurvival factor, SDSURVL and SDCASL2 expression was analyzed in sponge tissue and primmorphs challenged with proapoptotic stimulants. Moreover, the viability of mocktransfected and HEK293-SDSURVL cell lines was assessed after the exposure to the same stimuli. Pam ${ }_{3}$ Cys-Ser-(Lys) ${ }_{4}$ and cadmium were used for their selective ability to trigger extrinsic (the former compound) or intrinsic (latter) cell death pathways. ${ }^{22,23}$ Both lipopeptide and heavy metal stimulated the expression of SDSURVL and SDCASL2 in sponge tissue and primmorphs alike, already after $6 \mathrm{~h}$ (Figure 7). With prolonged incubation time, the expression level of both transcripts further increased; however, this effect was more pronounced in primmorphs than in tissue samples.

To assess putative effects of SDSURVL expression, mock-transfected HEK-293 and HEK293-SDSURVL cell lines $(95.5 \%$ transfection efficiency) were exposed to
Pam $_{3}$ Cys-Ser-(Lys) $)_{4}$ and cadmium. Then, 3-(4,5-dimethylthiazol-2-yl)-2,5-diphenyltetrazolium bromide (MTT) assays, cell death detection enzyme-linked immunosorbent assay (ELISA), and western blot analyses were carried out. MTT assays revealed that the lipopeptide did not alter cell viabilities, neither in mock-transfected nor in HEK293SDSURVL cells, compared with untreated control cells (Figure 8a). In addition, $\mathrm{Pam}_{3}$ Cys-Ser-(Lys) $)_{4}$ treatment did not change the amount of survivin (endogenous as well as poriferan). In contrast, both cell lines were sensitive to cadmium. Thus, MTT assays showed that $46.7 \%$ of mocktransfected cells underwent cell death after $12 \mathrm{~h}$ of exposure. Nevertheless, SDSURVL expression rescued HEK293SDSURVL, as only $25.6 \%$ cells were nonviable (Figure $8 \mathrm{~b}$; significance level $P \leqslant 0.05)$. Furthermore, western blot analyses showed an additional effect of cadmium, as increased immunodetection was observed of both, human survivin and its poriferan homolog after $6-12 \mathrm{~h}$ of challenge.

Concurrently, cell death detection ELISA could not reveal altered formation of mono- and oligonucleosomes, neither in mock-transfected nor HEK293-SDSURVL cells after $\mathrm{Pam}_{3}$ Cys-Ser-(Lys) $)_{4}$ treatment, compared with untreated control cells (Figure 9a). In contrast, exposure to cadmium significantly induced apoptotic DNA fragmentation in both cell lines. However, SDSURVL expression caused a reduced DNA fragmentation by $51 \%$ (Figure $9 \mathrm{~b}$; significance level $P \leqslant 0.05)$.

\section{Discussion}

Survivin represents a unique protein within the IAP family, containing a single BIR domain and a $\mathrm{COOH}$-terminal coiledcoil structure. Thus, it differs from all other family members that comprise 2-3 BIR domains and a canonical $\mathrm{COOH}$ terminal RING finger. ${ }^{11}$ In vertebrates, survivin not only controls cell cycle checkpoints and correct completion of cytokinesis but also regulates activation of caspases. ${ }^{24}$ However, the evolutionary conservation of this dual role 

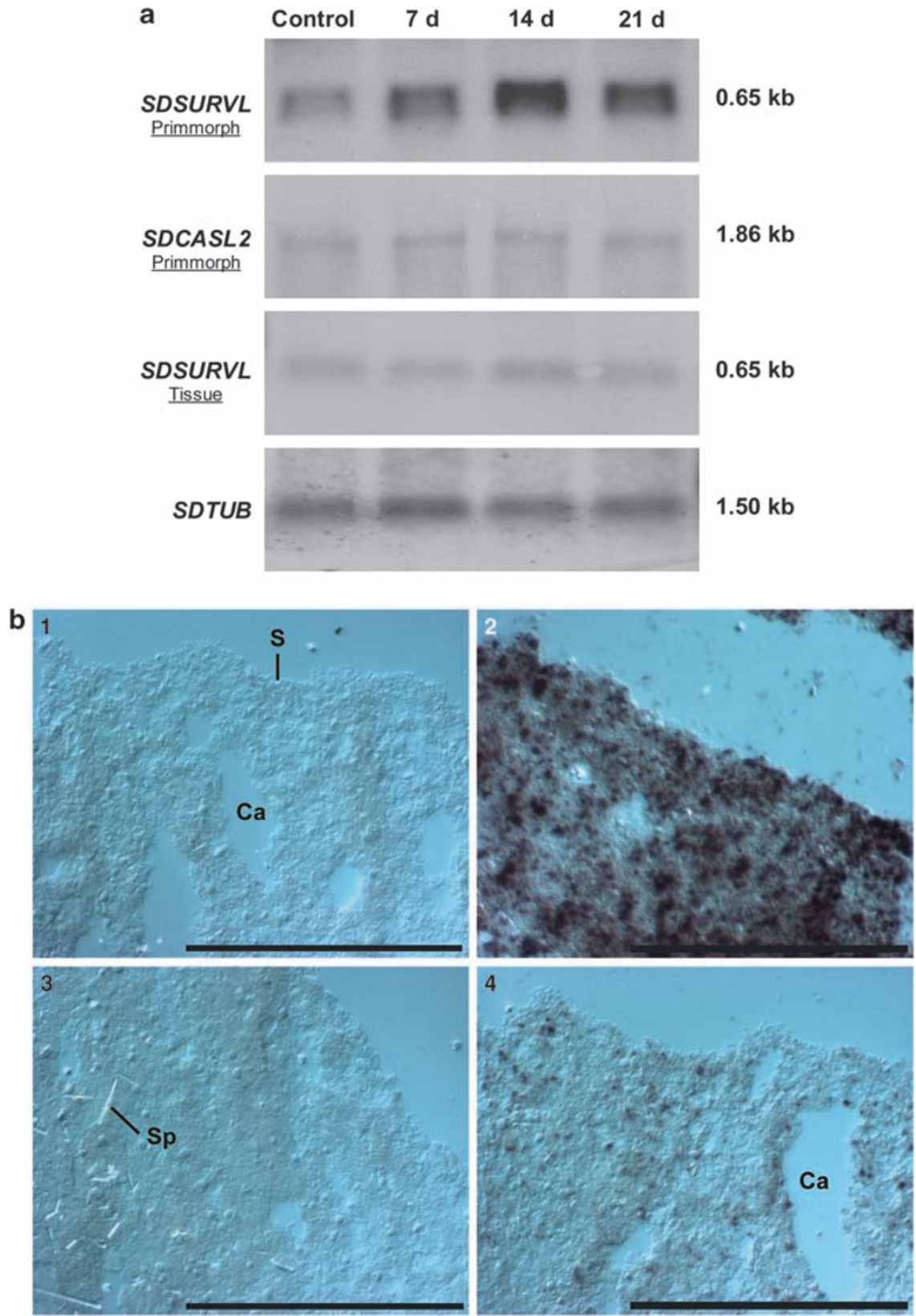

Figure 4 Expression of SDSURVL and SDCASL2 in Suberites domuncula primmorphs and adult tissue. (a) Northern blotting analyses. SDSURVL and SDCASL2 transcription was monitored in aging primmorphs and in adult sponge tissue (control, 7, 14, and 21 d). Tubulin transcription was used as an internal control. (b) In situ hybridization analyses. Cryosections of primmorphs (2 weeks old) were subjected to in situ hybridization analyses, using labeled SDSURVL $(1,2)$ or $\operatorname{SDCASL2}(3,4)$ probes $(1 / 3$, sense (negative control) or $2 / 4$, antisense probe). Hybridized probes were detected through anti-DIG Fab fragments, conjugated to alkaline phosphatase, and subsequent NBT/BCIP treatment. The sense probes $(1,3)$ did not produce any signal, whereas the SDSURVL antisense probe $(2)$ abundantly and homogenously detected transcripts. The SDCASL2 antisense probe (4) identified few transcripts in the surface cell layer (S) and in cells lining the aquiferous canal system (Ca) within the mesohyl, the internal part of the sponge. Sponge spicules $(\mathrm{Sp})$ are marked. $\mathrm{Bar}=1 \mathrm{~mm}$

remains inconclusive, as in Nematoda the survivin homolog seems to be implicated exclusively in cytokinesis. ${ }^{25}$ In addition, even though a fungal ( $S$. pombe) survivin-like protein was proposed to be required for efficient cell division and exhibits antiapoptotic activity, ${ }^{26}$ it is considerably larger in size than other survivin proteins.

Key regulators of apoptosis and cell cycle progression have already been discovered in the phylum Porifera, ${ }^{27}$ which seems to be the earliest-branching metazoan taxon. ${ }^{4}$ However, identification of poriferan survivin provides an important tool not only to investigate the primordial function(s) of this versatile regulator protein, but also to elucidate the putative ancient and intimate link between apoptosis, proliferation, and morphogenesis.

The newly discovered poriferan survivin homolog showed a significant degree of sequence conservation. A consensus 

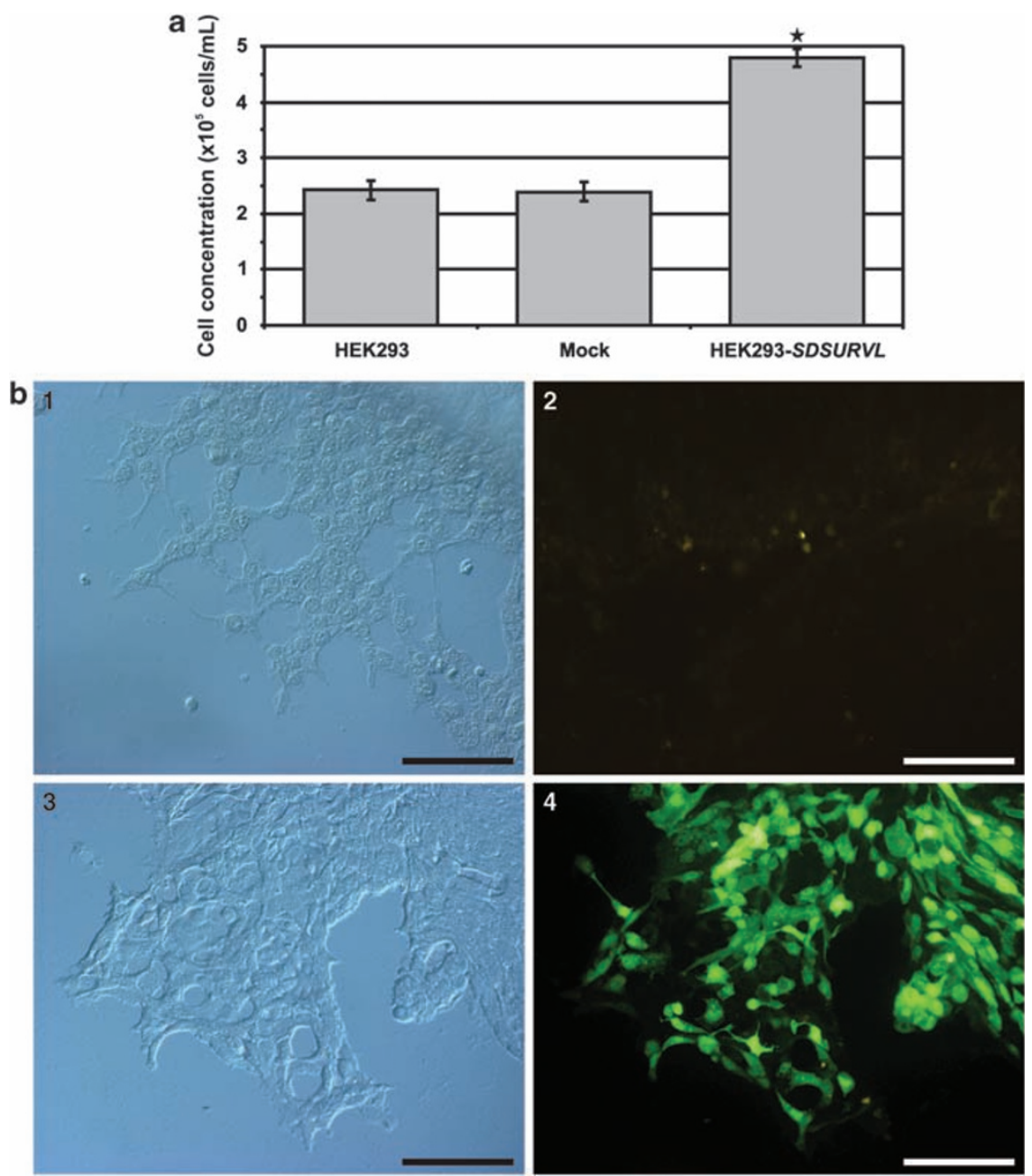

Figure 5 SDSURVL expression in HEK-293 cells. (a) Effect of SDSURVL expression on cell proliferation. Cells were transfected either with empty vector (Mock) or with a SDSURVL construct (HEK293-SDSURVL) or remained untransfected (HEK293). Cell concentrations were measured $48 \mathrm{~h}$ after transfection. Proliferation rates of Mock and HEK293 were comparable, showing that the vector itself had no effect. However, HEK293-SDSURVL revealed a significantly higher proliferation rate (the asterisk denotes the statistical significance, $P \leqslant 0.05$ ). Bars represent mean \pm S.D. of three independent experiments, each with $n=5$. (b) Histological detection of the SDSURVL-EGFP fusion protein in transfected HEK-293 cells. Mock-transfected HEK-293 cells $(1,2)$ and HEK293-SDSURVL $(3,4)$ under visible $(1 / 3)$ or UV light $(2 / 4)$. Bar $=100 \mu \mathrm{m}$

tree resulting of $\mathrm{NJ}, \mathrm{ML}$, and Bayesian analyses places SDSURVL at the base of a metazoan clade, confirming the common ancestry of metazoan survivin-like proteins, whereas viral sequences form a well-supported separate group.

SDSURVL features all characteristics of survivins that facilitate protein interaction and mediate both antiapoptotic activity and proper kinetochore attachment. ${ }^{28}$ However, it shows several additional putative domains that are known to be involved in cell cycle regulatory functions: (i) APCC-D box, required for ubiquitination and proteasome-mediated degradation by the anaphase-promoting ubiquitin ligase complex (APC/C); (ii) LIG_FHA1/2 motifs (aa $\left.a_{98-104} ; a_{100-106}\right)$ that bind regulators of the $G_{2} / M$ checkpoint; (iii) USP7-binding motif $\left(a_{33-37}\right)$ that is a target of the UPS7 deubiquitinating enzyme, whose substrates are regulators of cell survival pathways. So far, no such feature has been described for other survivin molecules.

The gene structure (intron/exon positions, intron phases) is conserved between poriferan and vertebrate (human, murine) survivin, ${ }^{18}$ but not within the $D$. melanogaster deterin,${ }^{13}$ which possesses only two introns. However, some differences to the vertebrate promoter region are noticeable: the TATA-less vertebrate promoter includes $\mathrm{CpG}$ islands and several $\mathrm{Sp} 1$, $\mathrm{CDE}$, and CHR sites. By analogy with other cell cycleregulated genes it was postulated that $S p 1$ regulates basal transcriptional expression of the survivin gene, further modulated by $\mathrm{CDE} / \mathrm{CHR}$ elements, thus imparting cell cycle periodicity of survivin expression. ${ }^{18}$ In contrast, the poriferan promoter features a canonical TATA box, two Sp1 sites, and is missing $\mathrm{CDE} / \mathrm{CHR}$ elements. Therefore, considering the aforementioned protein interaction motifs, regulation of the poriferan survivin pool might also occur on a posttranscriptional/proteasomal level, for example, by (de)ubiquitination or complex formation with other regulatory proteins.

Survivin is expressed in many human tumors and in rapidly dividing cells of fetal tissue during the $G_{2} / M$ phase, but is not detectable in terminally differentiated cells. It was proposed that survivin exerts its antiapoptotic function by inhibiting 

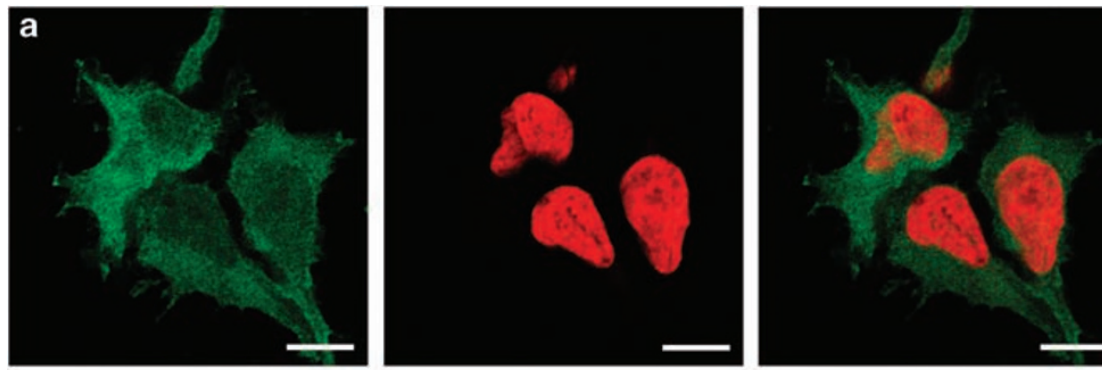

- LMB
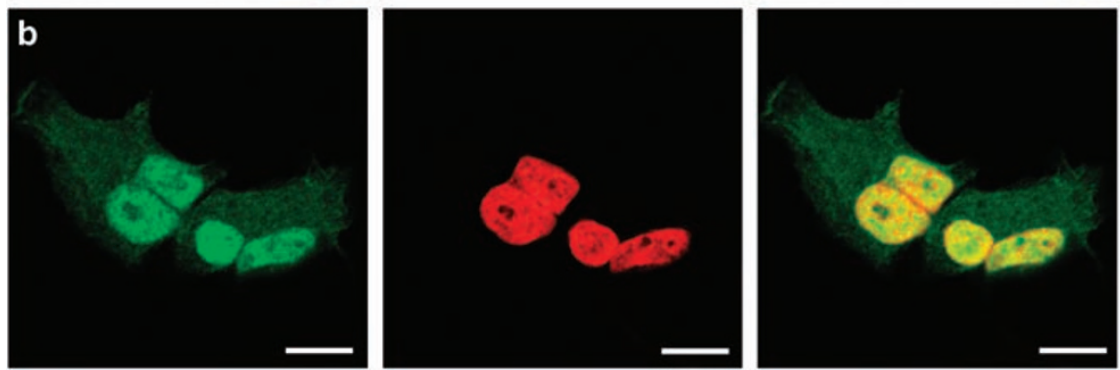

Figure 6 Subcellular localization of SDSURVL-EGFP in transfected HEK-293 cells. SDSURVL-EGFP expressing cells either remained untreated (a, control) or were incubated with the nuclear export inhibitor LMB $(6 \mathrm{nM}, 30 \mathrm{~min} ; \mathbf{b})$. The localization of the fusion protein (green) was analyzed by laser scanning microscopy. Nuclei were DRAQ5-stained (red). The merge pictures revealed a predominantly cytoplasmic localization of SDSURVL-EGFP in the control, whereas on LMB treatment the EGFP-tagged protein accumulated in the nucleus (yellow). The panels show representative examples of SDSURVL-EGFP expressing cells. Bars, $10 \mu \mathrm{m}$
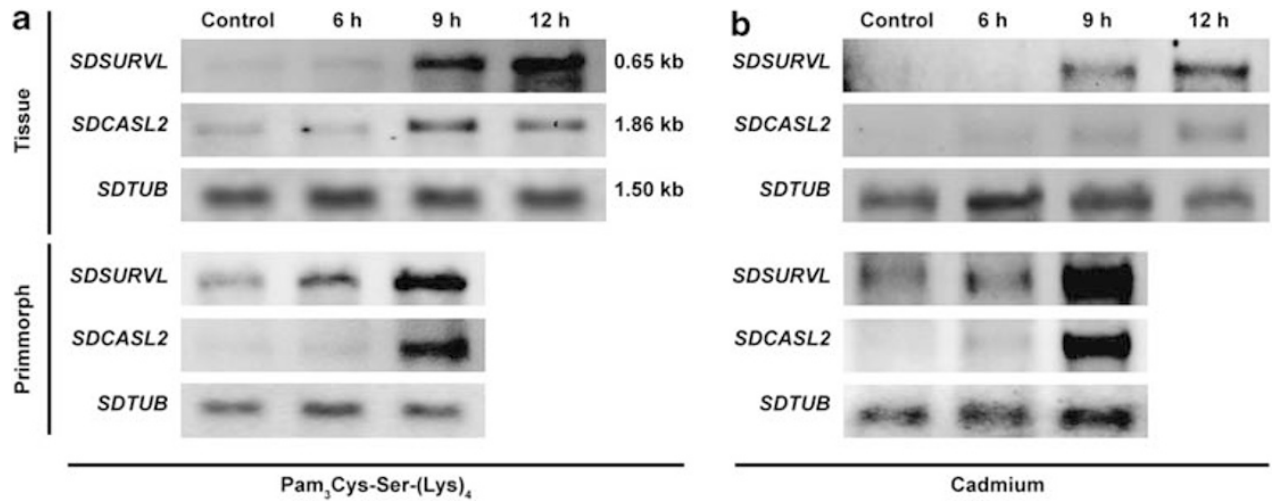

Figure 7 Gene expression analyses of challenged sponge tissue and primmorphs. Following exposure of sponge tissue and primmorphs to Pam ${ }_{3}$ Cys-Ser-(Lys) $)_{4}(\mathbf{a})$ or cadmium (b) for the indicated time, RNA was isolated, size separated (equal amounts of RNA were loaded), and blotted. The blots were probed for transcripts of poriferan survivin-like protein (SDSURVL), caspase-like protease 2 (SDCASL2), and tubulin (SDTUB). The latter one was used as an internal control

caspases, particularly caspase- 7 and $-3,{ }^{29}$ though other studies argue against a direct mechanism of caspase inhibition by survivin, see for example, Banks et al. ${ }^{30}$

To approach the enigmatic role of survivin in nonbilaterian Metazoa, comparative functional studies of poriferan survivin were conducted. Accordingly, the implementation of SDSURVL in cell proliferation and cell death was studied in sponges as well as in a human cell model (HEK-293; as SDSURVL-EGFP fusion). This strategy had already been successfully adopted to assess the antiapoptotic potential of deterin and poriferan B-cell lymphoma (Bcl)-2. ${ }^{31,32}$ Moreover, survivin-EGFP fusions have been widely used to study both the bifunctional role of survivin and its dynamic intracellular localization and seem fully functional. ${ }^{33-35}$ In contrast to sponge tissue that show a very low steadystate expression level, proliferating cells of primmorphs contained a considerable amount of SDSURVL, indicative of its involvement in cell division. This assumption was supported by a significantly increased proliferation rate of HEK-293 cells expressing SDSURVL-EGFP. In addition, application of two inducers of cell death (cadmium, $\mathrm{Pam}_{3}$ Cys-Ser-(Lys) $)_{4}$ ) indicated an antiapoptotic function of SDSURVL, as both compounds triggered the expression of SDSURVL in sponge tissue and primmorphs, possibly a countermeasure to the induced expression of the caspase-7 homolog SDCASL2. Concurrently, cadmium induced apoptosis in HEK-293 cells, although concomitant expression of SDSURVL-EGFP drastically reduced the occurrence of cell death $(\approx 50 \%)$. However, $\mathrm{Pam}_{3}$ Cys-Ser-(Lys) $)_{4}$ incubation did not alter HEK-293 cell viability, probably because of the different repertoire of Toll-like receptors (mediating lipopeptide-induced innate immunity and apoptosis) found 


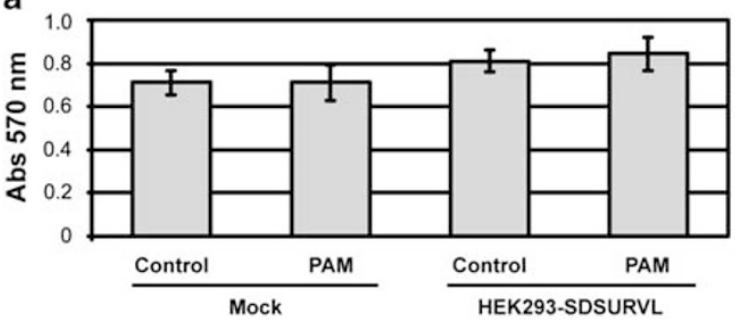

b

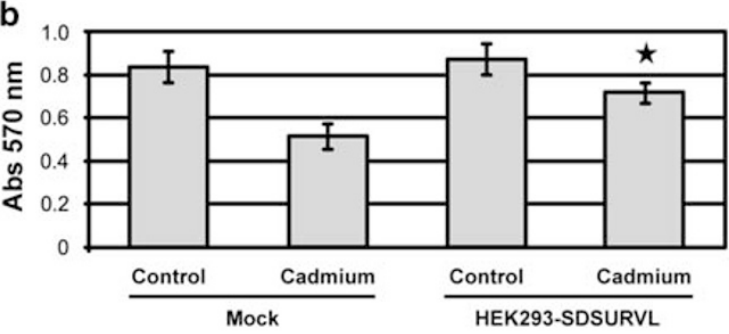

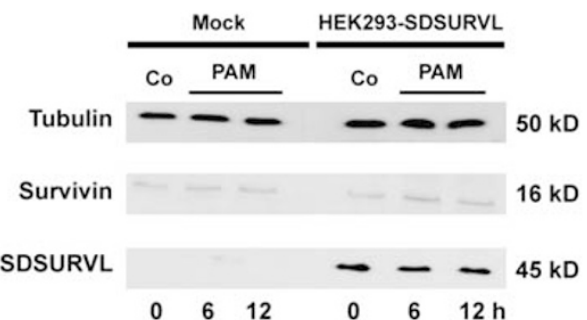

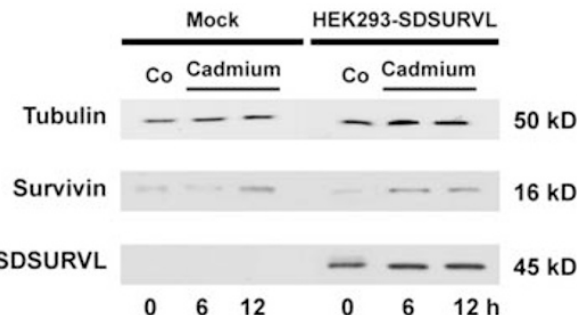

Figure 8 SDSURVL-EGFP-mediated effects on Pam ${ }_{3}$ Cys-Ser-(Lys) 4 - and cadmium challenged HEK-293 cells. Viability (MTT assays, left; bars represent mean \pm S.D. of three independent experiments, each with $n=5$ ) and protein detection (western blot analyses, right) of HEK293-SDSURVL and mock-transfected (Mock) cells, following

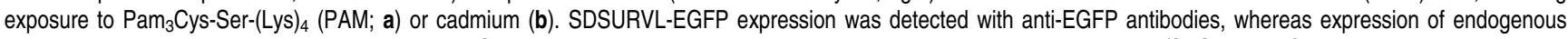
survivin was detected with anti-survivin antibodies. Co, untreated cells. Whereas PAM altered neither cell viability nor survivin/SDSURVL-EGFP concentrations, cadmium provoked significantly decreased cell viability. However, HEK293-SDSURVL cells were less sensitive to cadmium than mock-transfected controls (the asterisk denotes the statistical significance, $P \leqslant 0.05$ ) and showed an increased accumulation of both endogenous survivin and SDSURVL-EGFP

a

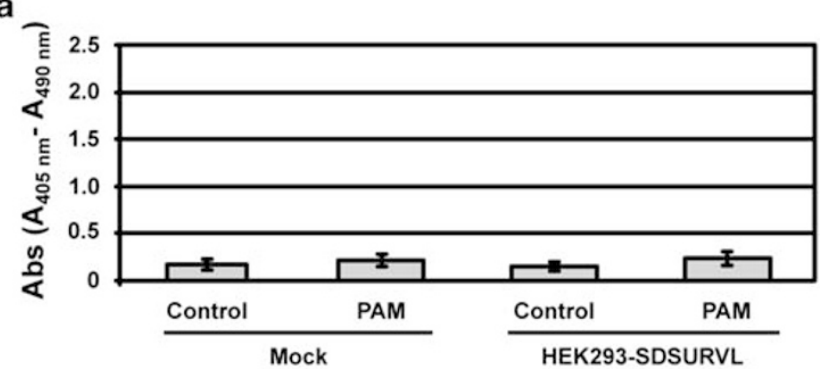

b

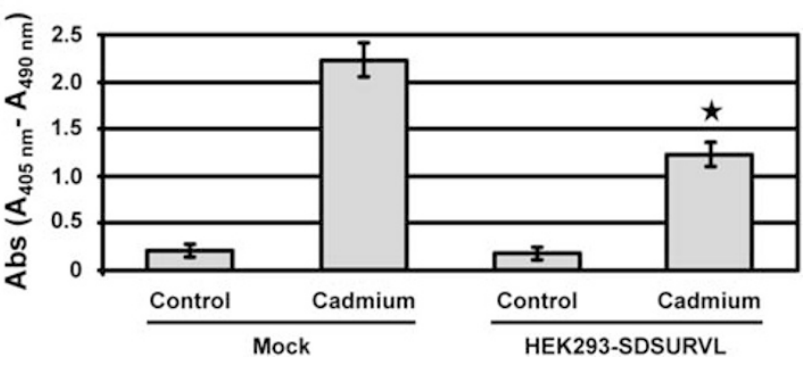

Figure 9 Susceptibility of HEK-293 cells to Pam ${ }_{3}$ Cys-Ser-(Lys) $)_{4}$ - and cadmium. Following exposure to $\mathrm{Pam}_{3}$ Cys-Ser-(Lys) (PAM; a) or cadmium (b), apoptotic fragmentation of genomic DNA was assessed immunologically in HEK293-SDSURVL and mock-transfected HEK-293 (Mock) cells. In this cell death detection ELISA, immobilized cytoplasmic histone-associated DNA fragments were detected through consecutive addition of peroxidase-conjugated anti-DNA antibodies and 2,2' -azino-bis (3-ethylbenzthiazoline-6-sulfonic acid and, then, quantified photometrically. Co, untreated cells. Data represent mean \pm S.D. of four independent experiments in triplicate. Whereas PAM did not cause internucleosomal fragmentation of genomic DNA (neither in HEK293SDSURVL nor in mock-transfected HEK-293), cadmium induced apoptotic degradation of DNA. However, expression of SDSURVL-EGFP significantly reduced this effect compared with mock-transfected controls (the asterisk denotes the statistical significance, $P \leqslant 0.05$ )

within HEK-293 and sponge cells. Future studies will investigate the effect of further inducers of cell death on sponge and HEK293-SDSURVL cells.
Cytotoxicity mediated by cadmium is associated with the release of mitochondrial proapoptotic factors into the cytosol. ${ }^{23}$ These factors cause activation of caspase- $3 / 7,{ }^{36}$ which is considered as a biochemical hallmark of the apoptotic cascade. However, survivin was proposed to interact with various caspases and, consequently, to inhibit further progression of the apoptotic cascade. The aforementioned observations in combination with significant similarities of sequence and structure between SDSURVL and its vertebrate counterparts propose a common regulatory function in the interconnected pathways of cell cycle and apoptosis. In HEK-293 cells, poriferan survivin possibly complements the endogenous molecule by sequestrating proapoptotic binding partners, consequently delaying cell death. In addition, the presence of putative target sites for ubiquitination might explain the elevated levels of human and poriferan survivin detected in challenged transfected cells. Accordingly, this effect is not the result of an induced expression but is indicative of a decreased proteasomal degradation of either protein. Therefore, the poriferan molecule replenishes the pool of endogenous survivin, concomitantly preventing degradation of its human counterpart by engaging/silencing elements of the proteasome.

Furthermore, concurrent with previous observations during which elevated levels of survivin occur in most human tumor cell types and overexpression of survivin in transfected HEK-293 increases cell proliferation, ${ }^{20,21}$ SDSURVL-EGFP expression stimulates cell division. In addition, LMB treatment of SDSURVL-EGFP expressing HEK-293 cells causes significant nuclear accumulation of the fusion protein. This is consistent with previous studies in which chromosome region maintenance 1-mediated, NES-dependent nuclear export of human survivin was blocked by LMB. ${ }^{10,16}$ Survivin is a subunit of the chromosomal passenger complex (CPC), consisting of Aurora-B, Borealin and INCENP, and is essential for proper 
chromosome segregation and cytokinesis. As Crm1 is involved in tethering the CPC to the centromere by interacting with survivin's NES, ${ }^{10}$ SDSURVL might affect various stages of cell proliferation, from proper kinetochore attachment to spindle formation.

In conclusion, the identification of a bona fide poriferan survivin homolog represents a compelling opportunity to investigate the deep evolutionary original function(s) of survivin, whereas concurrently avoiding limitations of classical invertebrate model organisms. The data presented propose an evolutionary ancient dual regulatory role of survivin, implemented in cell cycle control and programmed cell death.

\section{Materials and Methods}

Preparation of $\boldsymbol{S}$. domuncula tissue and primmorphs. Specimens of the marine sponge Suberites domuncula (Porifera, Demospongiae, Hadromerida) were collected near Rovinj (Croatia) in the northern Adriatic Sea and then kept in aquaria in Mainz (Germany) at $17^{\circ} \mathrm{C}$.

Primmorphs were obtained after tissue dissociation into single cells as described earlier. ${ }^{37}$ They were cultured in natural sterile-filtered seawater (Sigma-Aldrich, Taufkirchen, Germany), supplemented with $0.2 \%$ (v/v) RPMI-1640 medium (Biochrom AG, Berlin, Germany) and $60 \mu \mathrm{M}$ silicate. Cultures were shaken for 5 days to assist the formation of aggregates, consisting of proliferating cells. Then, two sets of experiments were performed. For the first set, primmorphs were exposed to cadmium ( $\mathrm{CdCl}_{2}$, Sigma-Aldrich; $50 \mu \mathrm{M}$ ) or the synthetic lipopeptide (S)-(2,3-bis(palmitoyloxy)-(2-RS)-propyl)-N-palmitoyl-(R)-Cys-(S)-Ser-(S)-Lys ${ }_{4}-\mathrm{OH}$. $3 \mathrm{HCl}\left(\mathrm{Pam}_{3}\right.$ Cys-Ser-(Lys) 4 ; Axxora; Grünberg, Germany; $\left.40 \mu \mathrm{g} / \mathrm{ml}\right)$ for 6 and $9 \mathrm{~h}$, or they remained untreated as a control. For the second set of experiments, samples were taken 0 (control), 7, 14, and 21 days after primmorph formation. All experiments were performed under mild shaking.

In a similar way, $S$. domuncula tissue was exposed to cadmium $(50 \mu \mathrm{M})$ or $\mathrm{Pam}_{3}$ Cys-Ser-(Lys) ${ }_{4}(40 \mu \mathrm{g} / \mathrm{ml})$ for up to $12 \mathrm{~h}$.

RNA extraction and northern blotting analyses. Total RNA was isolated through lysis of homogenized tissue/primmorphs using TRIzol Reagent (Invitrogen, Karlsruhe, Germany) as described earlier. ${ }^{38}$ Subsequently, $5 \mu \mathrm{g}$ of total RNA was size separated and blotted on Hybond N1 membranes (Amersham, Freiburg, Germany). Labeled probes were generated with DIG-11-deoxyuridine triphosphate through the PCR-DIG-Probe Synthesis Kit (according to the manufacturer's instructions (Roche Applied Science, Mannheim, Germany)). Hybridization was carried out with a $S$. domuncula survivin-like probe (SDSURVL, $\mathrm{nt}_{1-357}$ ) or a caspase-like protease 2 probe $\left(S d C A S L 2, \mathrm{nt}_{810-1480}\right)$. Furthermore, a probe was designed to detect the expression of the housekeeping gene $\beta$-tubulin (SDTUB, $\mathrm{nt}_{83-483}$; NCBI accession number AJ550806) as internal reference. Hybridized probes were detected with anti-DIG Fab fragments (conjugated to alkaline phosphatase) and ultimately visualized by chemiluminescence, using the 1,2-dioxetane compound CDP Star (Roche) as substrate.

Cloning of $\mathbf{S}$. domuncula survivin-like protein and caspase-like protease 2. cDNA coding for the survivin-like protein SDSURVL was isolated from a $S$. domuncula $C D N A$ library ${ }^{39}$ by PCR with degenerate primers that were designed against the conserved BIR domain (forward primer, $5^{\prime}$-GAT GGC IGA GGC T/CGG CTT-3'; reverse primmer, 5'-CT/CA TGT CCA GTT TT/CA AG/AA-3'; where $\mathrm{I}=$ inosine). $\mathrm{PCR}$ was carried out at an initial denaturation at $95^{\circ} \mathrm{C}$ for $5 \mathrm{~min}$ followed by 35 amplification cycles at $95^{\circ} \mathrm{C}$ for $30 \mathrm{~s}, 54^{\circ} \mathrm{C}$ for $45 \mathrm{~s}$, and $72^{\circ} \mathrm{C}$ for $1 \mathrm{~min}$, and terminated with a final extension at $72^{\circ} \mathrm{C}$ for $10 \mathrm{~min}$. Thus, a fragment of $\approx 209 \mathrm{bp}$ was isolated and subsequently sequenced using an automatic DNA sequencer (Li-Cor 4300, Li-Cor, Bad Homburg, Germany). Ultimately, the SDSURVL sequence was completed through primer walking. Subsequently, genomic sequences were extracted from a $S$. domuncula genomic DNA library. ${ }^{40}$

Similarly, SDCASL2 was isolated from the $S$. domuncula CDNA library: application of the degenerate forward primer $5^{\prime}-\mathrm{T} / \mathrm{CTI}$ ATT/C/A CAA/G GCI TGT/C A/T/CG/TI GG-3' (directed against the caspase Cys active site L/F-I-D-A-C-R/L-G) combined with a library-specific primer resulted in a fragment of $\approx 1200 \mathrm{bp}$. PCR was carried out at an initial denaturation at $95^{\circ} \mathrm{C}$ for $5 \mathrm{~min}$, followed by 35 amplification cycles at $95^{\circ} \mathrm{C}$ for $30 \mathrm{~s}, 54^{\circ} \mathrm{C}$ for $45 \mathrm{~s}$, and $72^{\circ} \mathrm{C}$ for $2 \mathrm{~min}$, and terminated with a final extension at $72^{\circ} \mathrm{C}$ for $10 \mathrm{~min}$. The complete cDNA was isolated by using a combination of sequence- and library-specific primers.

Sequence analyses. Putative promoter regions were analyzed with TESS (http://www.cbil.upenn.edu/cgi-bin/tess/tess?), TFSEARCH (http://www.cbrc.jp/research/ $\mathrm{db} /$ TFSEARCH), TATA Signal Prediction in Eukaryotic Genes (http://zeus2.itb.cnr. it/ webgene/wwwHC_tata.html), and CpGProD (http://pbil.univ-lyon1.fr/software/ cpgprod_query.html). Coiled-coil conformations were studied using COILS (http:// www.ch.embnet.org/software/COILS_form.html). Homology searches were performed via the servers at the European Bioinformatics Institute (Hinxton, UK (http://www.ebi. ac.uk/inc/head.html)) and the National Center for Biotechnology Information (NCBI, Bethesda, MD, USA (http://www.ncbi.nlm.nih.gov/BLAST/)). Phylogenetic and molecular evolutionary analyses were conducted using MEGA version 4 for $\mathrm{NJ}$ analyses or were obtained through the phylogeny.fr server (http:/www.phylogeny.fr/version2_cgi/ programs.cgi) for ML analyses and Bayesian inference analyses (MRBAYES). ${ }^{41,42}$ For NJ and ML analyses the degree of support of internal branches was assessed by bootstrapping. For Bayesian analyses posterior probability values were estimated. Potential subunits, domains, patterns, and transmembrane regions were predicted after searching databases of Pfam (http://www.sanger.ac.uk/Software/Pfam/), SMART (http://smart.embl-heidelberg.de/), and ELM (http://www.elm.eu.org/)

Vector constructs, transfections, and LMB treatment. The forward primer $5^{\prime}$-GAA TTC ATG GCT AAT ACG TAT GAG A-3' (EcoRl site is underlined) and the reverse primer $5^{\prime}$-GGA TCC CGA TGT TCG TGT CTT TTC A-3' (BamHI) were used to amplify the ORF of SDSURVL ( $\mathrm{nt}_{1-447}$ ), including $\mathrm{M}_{\text {start }}$ and excluding the first stop codon. The CDNA was ligated in frame into the eukaryotic expression vector pEGFP-N2 (Clontech, Heidelberg, Germany). The recombinant protein, thus, contained a $\mathrm{COOH}$-terminal EGFP-tag.

Human embryonic kidney (HEK-293; ATCC CRL-1573) cells were obtained from the American Type Culture Collection (LGC Promochem, Wesel, Germany) and grown in Dulbecco's Modified Eagle Medium (high glucose and L-glutamine; Gibco, Karlsruhe, Germany) with $10 \%$ (v/v) fetal bovine serum. Cell counting was performed using a Neubauer chamber and trypan blue staining, three times for each sample.

On the eve of transfection, cells were seeded to allow for $\approx 60 \%$ confluency on the next day. The transfection technology was provided by IBA (Göttingen, Germany) and based on the combination of cationic liposomes and magnetic DNA-binding nanoparticles. Transfections were carried out in 12-wells plates with $1.2 \mu \mathrm{g}$ DNA per well. For constitutive expression, the cells were split $48 \mathrm{~h}$ after transfection into a selective medium, containing G418 at the appropriate concentration (Carl Roth, Karlsruhe, Germany; $200 \mu \mathrm{g} / \mathrm{ml}$ ). SDSURVL expression was confirmed microscopically and immunologically, using anti-EGFP antibodies (Miltenyi Biotec GmbH, Bergisch Gladbach, Germany), and an AHBT3 light microscope with AH3-RFC reflected light fluorescence attachment (Olympus, Hamburg, Germany).

The nucleocytoplasmic trafficking of the SDSURVL fusion protein was analyzed through treatment of transfected cells with the nuclear export inhibitor LMB, according to. ${ }^{16}$ In short, $48 \mathrm{~h}$ after transfection cells were treated with LMB $(6 \mathrm{nM}$, $30 \mathrm{~min}$ ), washed in phosphate-buffered saline (PBS), and fixed in $4 \%(\mathrm{w} / \mathrm{v})$ paraformaldehyde (PFA; 5 min, RT). After additional washing of cells with PBS and subsequent counterstaining of nuclei with DRAQ5 $(5 \mu \mathrm{M}$; Biostatus Ltd., Shepshed, UK), images were acquired on a Zeiss 710 confocal LSM (Zeiss, Göttingen, Germany). The argon laser line of $488 \mathrm{~nm}$ was used to excite EGFP and a $633 \mathrm{~nm}$ HeNe laser was used to excite DRAQ5. For illustration purposes, green and red channels were extracted from the merged pictures with the ZEN software (Zeiss).

In situ localization studies. The in situ hybridization method applied was based on a procedure described by Wiens et al. ${ }^{39}$ In short, frozen sections $(8 \mu \mathrm{m})$ of primmorph samples were fixed in $4 \%$ paraformaldehyde, then incubated with proteinase $\mathrm{K}(1 \mu \mathrm{g} / \mathrm{ml})$, and fixed again in paraformaldehyde. Hybridization was carried out with single-stranded SDSURVL DNA probes, overnight at $45^{\circ} \mathrm{C}$ in $2 \times$ SSC (sodium chloride/sodium citrate, supplemented with $50 \%$ formamide). The probes (236 nt each) were generate during two separate PCR reactions, either with forward primer $5^{\prime}$-TAT GAG AGT TGT GAC AGA GTA AA-3' (sense/control probe) or with reverse primer $5^{\prime}$-TGT TCA TCA CGG GGA TTG TCT GA-3' (antisense probe), using SDSURVL CDNA as template. During the linear amplification the probes were labeled with the DIG-Probe Synthesis Kit (Roche Applied Science, Mannheim, Germany). In a similar way SDCASL2 probes (292 nt) were obtained, using forward primer $5^{\prime}$-GGT GTG TGG CTT ACA AAA CTT TC-3' 
or reverse primer $5^{\prime}$-GAA CAG ACT CAG TTG GAG ATG CT-3'. Following hybridization the sections were washed at $50^{\circ} \mathrm{C}$ at decreasing salt concentrations $(1 \times-0.2 \times$ SSC), blocked, and then incubated with anti-DIG Fab fragments, conjugated to alkaline phosphatase. Through addition of NBT and BCIP hybridized probes were visualized.

Western blot analyses. Mock-transfected (Mock) and SDSURVL-expressing (HEK293-SDSURVL) cells were exposed to Pam ${ }_{3}$ Cys-Ser-(Lys) ${ }_{4}(40 \mu \mathrm{g} / \mathrm{ml}, 6$ and $12 \mathrm{~h}$ ), or cadmium ( $\mathrm{CdCl}_{2}, 50 \mu \mathrm{M}, 6$, and $12 \mathrm{~h}$ ). Cells were then lysed with an adequate volume of NP40 lysis buffer $(50 \mathrm{mM}$ Tris- $\mathrm{HCl}, 150 \mathrm{mM} \mathrm{NaCl}, 1 \%(\mathrm{v} / \mathrm{v})$ NP-40, pH 8.0; 20 min on ice). After centrifugation, the total protein concentration was determined in the supernatant with BCA Protein Assay Reagent (Pierce, Rockford, IL, USA), according to the manufacturer's protocol. A volume of $20 \mu \mathrm{g}$ of total proteins were subjected to electrophoresis through $14 \%$ (v/v) polyacrylamide gels, containing $0.1 \%(\mathrm{w} / \mathrm{v})$ SDS. Following electroblotting, PVDF membranes (Invitrogen) were incubated with rabbit anti-EGFP (1:1000), conjugated with horseradish peroxidase. Immune complexes were visualized with Supersignal West Pico Chemiluminescent substrate (Pierce) on X-ray films. Other antibodies were acquired from Serotec (Raleigh, NC, USA), including anti-survivin (1:500; directed against $a_{1-12}$ of human survivin, a region without homology to SDSURVL) and anti-tubulin (1:1000; human)

MTT assays and cell death detection ELISA. In MTT assays the activity of mitochondrial succinate dehydrogenase is measured as indicator of cellular metabolic rate and viability. To determine the effect of SDSURVL expression, transfected and mock-transfected HEK-293 cells were seeded into 96-well plates $\left(0.5 \times 10^{4} \mathrm{celll} / \mathrm{ml}\right)$ and exposed to Pam $_{3}$ Cys-Ser-(Lys) $)_{4}(40 \mu \mathrm{g} / \mathrm{ml}, 12 \mathrm{~h})$ or cadmium $(50 \mu \mathrm{M}, 12 \mathrm{~h})$. Afterwards, $10 \mu \mathrm{l}$ of an MTT solution (Sigma-Aldrich, $5 \mathrm{mg} / \mathrm{ml}$ in PBS) were added to each well for $4 \mathrm{~h}\left(37^{\circ} \mathrm{C}\right)$. The resulting formazan crystals were dissolved in a stop solution ( $40 \%$ (v/v) dimethylformamide, $10 \%(\mathrm{w} / \mathrm{v})$ SDS). Absorbances were determined at $570 \mathrm{~nm}$ with a Bio-Rad 3550 ELISA plate reader (München, Germany).

In addition, the apoptotic response was measured in HEK293-SDSURVL and mock-transfected HEK-293 cells. As fragmentation of genomic DNA represents a hallmark of apoptosis, a cell death detection ELISA was applied, based on the immunochemical detection of mono- and oligonucleosomes in the cytoplasmic fractions of cell lysates. For this purpose, lysates of cells that had been exposed to Pam $_{3}$ Cys-Ser-(Lys) $)_{4}(40 \mu \mathrm{g} / \mathrm{ml}, 12 \mathrm{~h})$ or cadmium $(50 \mu \mathrm{M}, 12 \mathrm{~h})$ were transferred to streptavidin-coated 96-well plates and incubated with biotin-labeled anti-histone and peroxidase-conjugated anti-DNA antibodies, according to the manufacturer's instructions (Roche). Following extensive washing, immobilized antibody-histone complexes were visualized colorimetrically through incubation with peroxidase substrate 2,2'-azino-bis(3-ethylbenzthiazoline-6-sulfonic acid. Finally, absorbances were determined at 405 and $490 \mathrm{~nm}$ (reference).

Statistical analyses. Statistical analyses were performed with a Mann-Whitney U-test, a nonparametric statistical test for assessing whether the difference in medians between two samples is statistically significant. The level of significance was set at $P \leqslant 0.05$.

\section{Conflict of interest}

The authors declare no conflict of interest.

Acknowledgements. We thank $F$ Natalio for excellent technical assistance and CU Pietrzik for access to LSM. This work was supported by grants from the Deutsche Forschungsgemeinschaft (WI 2116/2-2) and the European Commission's Marie Curie Research Training Network 'BIOCAPITAL'.

1. Hengartner MO. The biochemistry of apoptosis. Nature 2000; 407: 770-776.

2. den Hollander Al, ten Brink JB, de Kok YJ, van Soest S, van den Born LI, van Driel MA et al. Mutations in a human homologue of Drosophila crumbs cause retinitis pigmentosa (RP12). Nat Genet 1999; 23: 217-221.

3. Bessou C, Giugia JB, Franks CJ, Holden-Dye L, Ségalat L. Mutations in the Caenorhabditis elegans dystrophin-like gene dys-1 lead to hyperactivity and suggest a link with cholinergic transmission. Neurogenetics 1998; 2: 61-72.
4. Philippe $H$, Derelle $R$, Lopez $P$, Pick $K$, Borchiellini $C$, Boury-Esnault $N$ et al Phylogenomics revives traditional views on deep animal relationships. Curr Biol 2009; 19: 706-712.

5. Love GD, Grosjean E, Stalvies C, Fike DA, Grotzinger JP, Bradley AS et al. Fossil steroids record the appearance of Demospongiae during the Cryogenian period. Nature 2009; 457 718-721.

6. Peterson KJ, Cotton JA, Gehling JG, Pisani D. The Ediacaran emergence of bilaterians: congruence between the genetic and the geological fossil records. Philos Trans $R$ Soc Lond B Biol Sci 2008; 363: 1435-1443.

7. Müller WEG, Schröder HC, Skorokhod A, Bünz C, Müller IM, Grebenjuk VA. Contribution of sponge genes to unravel the genome of the hypothetical ancestor of Metazoa (Urmetazoa). Gene 2001; 276: 161-173.

8. Gamulin V, Müller IM, Müller WEG. Sponge proteins are more similar to those of Homo sapiens than to Caenorhabditis elegans. Biol J Linn Soc 2000; 71: 821-828.

9. Kortschak RD, Samuel G, Saint R, Miller DJ. EST analysis of the cnidarian Acropora millepora reveals extensive gene loss and rapid sequence divergence in the model invertebrates. Curr Biol 2003; 13: 2190-2195.

10. Knauer SK, Mann W, Stauber RH. Survivin's dual role: an export's view. Cell Cycle 2007; 6 518-521.

11. Ambrosini G, Adida C, Altieri DC. A novel anti-apoptosis gene, BIRC5, expressed in cancer and lymphoma. Nat Med 1997; 3: 917-921.

12. Caldas $\mathrm{H}$, Jiang $\mathrm{Y}$, Holloway MP, Fangusaro J, Mahotka $\mathrm{C}$, Conway EM et al. Survivin splice variants regulate the balance between proliferation and cell death. Oncogene 2005; 24: 1994-2007.

13. Jones $G$, Jones $D$, Zhou $L$, Steller $H$, Chu Y. Deterin, a new inhibitor of apoptosis from Drosophila melanogaster. J Biol Chem 2000; 275: 22157-22165.

14. Fraser AG, James C, Evan GI, Hengartner MO. Caenorhabditis elegans inhibitor of apoptosis protein (IAP) homologue BIR-1 plays a conserved role in cytokinesis. Curr Biol 1999; 9: 292-301.

15. Coligan JE, Dunn BM, Ploegh HL, Speicher DW, Wingfield PT. Induction of immune responses In: Coligan JE (ed). Current Protocols in Protein Science. John Wiley \& Sons: New York, 2000, pp 2.0.1-2.8.17.

16. Heger P, Lohmaier J, Schneider G, Schweimer K, Stauber RH. Qualitative highly divergent nuclear export signals can regulate export by the competition for transport cofactors in vivo. Traffic 2001; 2: 544-555.

17. Wall NR, O'Connor DS, Plescia J, Pommier Y, Altieri DC. Suppression of survivin phosphorylation on Thr34 by flavopiridol enhances tumor cell apoptosis. Cancer Res 2003 63: $230-235$

18. Li F, Altieri DC. Transcriptional analysis of human survivin gene expression. Biochem $J$ 1999; 2: 305-311

19. Yuan J, Horvitz HR. A first insight into the molecular mechanisms of apoptosis. Cell 2004 116: 53-59.

20. Tamm I, Wang Y, Sausville E, Scudiero DA, Vigna N, Oltersdorf T et al. IAP-family protein survivin inhibits caspase activity and apoptosis induced by Fas (CD95), Bax, caspases, and anticancer drugs. Cancer Res 1998; 58: 5315-5320.

21. Torres VA, Tapia JC, Rodríguez DA, Párraga M, Lisboa $P$, Montoya $M$ et al. Caveolin-1 controls cell proliferation and cell death by suppressing expression of the inhibitor of apoptosis protein survivin. J Cell Sci 2006; 119: 1812-1823.

22. Aliprantis AO, Yang RB, Weiss DS, Godowski P, Zychlinsky A. The apoptotic signaling pathway activated by Toll-like receptor-2. EMBO J 2000; 19: 3325-3336.

23. Mao WP, Ye JL, Guan ZB, Zhao JM, Zhang C, Zhang NN et al. Cadmium induces apoptosis in human embryonic kidney (HEK) 293 cells by caspase-dependent and -independent pathways acting on mitochondria. Toxicol In Vitro 2007; 21: 343-354

24. Zangemeister-Wittke U, Simon HU. An IAP in action: the multiple roles of survivin in differentiation, immunity and malignancy. Cell Cycle 2004; 9: 1121-1123.

25. Li F. Survivin study: what is the next wave? J Cell Physiol 2003; 197: 8-29.

26. Uren AG, Beilharz T, O'Connell MJ, Bugg SJ, van Driel R, Vaux DL et al. Role for yeast inhibitor of apoptosis (IAP)-like proteins in cell division. Proc Natl Acad Sci USA 1999; 96 10170-10175.

27. Wiens M, Müller WEG. Cell death in Porifera: molecular players in the game of apoptotic cell death in living fossils. Can J Zool 2006; 84: 307-321.

28. Johnson AL, Langer JS, Bridgham JT. Survivin as a cell cycle-related and antiapoptotic protein in granulosa cells. Endocrinology 2002; 143: 3405-3413

29. Shin S, Sung BJ, Cho YS, Kim HJ, Ha NC, Hwang Jl et al. An anti-apoptotic protein human survivin is a direct inhibitor of caspase-3 and -7. Biochemistry 2001; 40: 1117-1123.

30. Banks DP, Plescia J, Altieri DC, Chen J, Rosenberg SH, Zhang $\mathrm{H}$ et al. Survivin does not inhibit caspase-3 activity. Blood 2000; 96: 4002-4003

31. Hawkins CJ, Ekert PG, Uren AG, Holmgreen SP, Vaux DL. Anti-apoptotic potential of insect cellular and viral IAPs in mammalian cells. Cell Death Differ 1998; 5: 569-576.

32. Wiens M, Diehl-Seifert B, Müller WEG. Sponge Bcl-2 homologous protein (BHP2-GC) confers distinct stress resistance to human HEK-293 cells. Cell Death Differ 2001; 8 887-898.

33. Delacour-Larose M, Molla A, Skoufias DA, Margolis RL, Dimitrov S. Distinct dynamics of Aurora B and Survivin during mitosis. Cell Cycle 2004; 3: 1418-1426.

34. Colnaghi R, Connell CM, Barrett RM, Wheatley SP. Separating the anti-apoptotic and mitotic roles of survivin. J Biol Chem 2006; 281: 33450-33456. 
35. Mahotka C, Liebmann J, Wenzel M, Suschek CV, Schmitt M, Gabbert HE et al. Differential subcellular localization of functionally divergent survivin splice variants. Cell Death Differ 2002; 9: 1334-1342.

36. Ozben T. Oxidative stress and apoptosis: impact on cancer therapy. J Pharm Sci 2007; 96 2181-2196.

37. Custodio MR, Prokic I, Steffen R, Koziol C, Borojevic R, Brümmer F et al. Primmorphs generated from dissociated cells of the sponge Suberites domuncula: a model system for studies of cell proliferation and cell death. Mech Ageing Dev 1998; 105: 45-59.

38. Grebenjuk VA, Kuusksalu A, Kelve M, Schütze J, Schröder HC, Müller WEG. Induction of $\left(2^{\prime}-5^{\prime}\right)$ oligoadenylate synthetase in the marine sponges Suberites domuncula and Geodia cydonium by the bacterial endotoxin lipopolysaccharide. Eur J Biochem 2002; 269: 1382-1392.
39. Wiens M, Korzhev M, Peroviæ-Ottstadt S, Luthringer B, Brandt D, Klein S et al. Toll-like receptors are part of the innate immune defense system of sponges (Demospongiae: Porifera). Mol Biol Evol 2007; 24: 792-804.

40. Seack J, Perovic S, Gamulin V, Schröder HC, Beutelmann P, Müller IM et al. Identification of highly conserved genes: SNZ and SNO in the marine sponge Suberites domuncula: their gene structure and promoter activity in mammalian cells. Biochim Biophys Acta 2001; 1520: 21-34.

41. Yang Z. Maximum likelihood phylogenetic estimation from DNA sequences with variable rates over sites: approximate methods. J Mol Evol 1994; 39: 306-314.

42. Huelsenbeck JP, Ronquist F. MRBAYES: Bayesian inference of phylogenetic trees. Bioinformatics 2001; 17: 754-755. 